\title{
The Case Study of Q Company Leadership Silence
}

\author{
Qiuying Wang *
}

\author{
School of Economic and Management, Beijing Jiaotong University, China, 100044 \\ *Corresponding author. Email: wangqiuying5752@163.com
}

\begin{abstract}
The phenomenon of silence is widespread in organizations, but at present, the research on organizational silence is mostly focused on the employee level, and rarely involves the leadership level. Q company is chosen as the case study object. Through the semi-structured interview method, this paper summarizes the influence results of leadership silence, explores the factors that affect leadership silence, and comprehensively and deeply understands leadership silence. Through the analysis of interview data, it is found that leaders' management experience, ability level, employee maturity and problematic nature can affect leadership silence. Leadership silence can not only serve to establish leadership authority, maintain organizational harmony and reduce personnel changes in enterprises, but also induce negative emotions of subordinates and affect the relationship between superiors and subordinates.
\end{abstract}

Keywords: Leader, Leadership silence, Influencing factors, Influencing results.

\section{INTRODUCTION}

Under the background of our traditional culture, people regard silence as the best choice to avoid conflict and maintain relations, and the phenomenon of silence is pervasive in the organization. At present, most of the researches concentrate on the discourse of the silence at the employee layer. Considering the different roles and status of leaders and employees in the organization, Huang Gui, a Chinese scholar, first put forward the concept of leadership silence and made a preliminary exploration [1].

Founded in 2004, Q Company is a business platform for entrepreneurs interested in the insurance intermediary service industry. It is an insurance agency with large scale, many institutions and comprehensive coverage in the Shandong insurance intermediary industry. Taking the Q Company as an example, this paper deeply explores the influencing results and influencing factors of leadership silence through indepth interviews with leaders and employees, puts forward suggestions for leaders to better use silence strategy, and promotes the sustainable, stable and healthy development of Q company.

\section{LITERATURE REVIEW}

Because leadership silence has profound cultural origin in our country and was first set forward by Chinese scholars, there are few works on leadership silence abroad. But in the organization silence research aspect, academia has obtained the rich results. Organizational silence was first raised by Morrison and Milliken and was considered as a collective phenomenon [2]. Pinder and Harlos believe that organizational silence refers to the ability of employees to change their current organizational environment while retaining an assessment of their emotion, cognition, and behavior [3]. They divide employee silence into tacit silence and inaction silence according to the response of employees to unfairness in the organization [3]. Dyne and others believe that organizational silence is the behavior of employees who deliberately retain their views or ideas on the improvement of their organization or work. They divide employees into active and passive according to their emotional characteristics, and then divide employee silence into pro-social silence, defensive silence and tacit silence [4].

At present, the work of leadership silence in China is nevertheless in its infancy, and only preliminary exploration has been borne away. Huang Gui puts 
forward the concept of leadership silence for the first time, defining it as the phenomenon or behavior of the leader of the organization who consciously does not clearly state his views and intentions in the process of formal communication, or deliberately reserved during the process of expression[1]. Liu Jun and others believe that leadership silence refers to the phenomenon or behavior in which leaders can change the current situation of the organization by communicating with employees but remain silent [5]. Through qualitative research, Huang Gui summed up twelve motivation of leadership silence and summed up six dimensions accordingly [6]. With the help of questionnaire design and data collection, leadership silence is divided into five dimensions, which are different from the conclusion of Huang Gui's qualitative research [7].

\section{CASE DESCRIPTION}

This study selected 6 leaders and 4 employees of the Q Company as interview objects. Through semistructured interviews with each of them, the general existence of silence of Q company leaders was verified. This paper selects two typical events of leadership silence to describe in detail, which are as follows.

\subsection{Event one-Implementation of the New Policy}

Q chairman's office, sales manager Wang and planning manager Liu were split up over the issuance of a new policy. Wang proposed to implement preferential policies for member customers in the company, while Liu firmly opposed considering the decline of the company's profits in recent years. In order to convince each other, the two chattered in the chairman's office. At this time the chairman remained silent, neither supporting Wang nor responding to Liu. After debating for a while, the two managers withdrew from the office.

However, the matter must be resolved. Wang took the initiative to contact Liu in the evening and made a detailed analysis to him. The two finally reached a settlement and jointly improved the policy, getting a new plan. The following morning, Wang and Liu came to the chairman's office together. Just opened the door, the chairman smiled and joked," Have you two figured it out and prepared to fool me together? "Mr. Wang first explained," No, we went back and analyzed. I said something wrong at that time, some factors were not taken into account, and manager Liu also said something imperfect. From the point of view of the matter itself, we worked out an optimal plan, so that the success rate will be higher ". Liu also said that he need to strengthen the learning in business. The chairman smiled with satisfaction and said," That's what I want. Next time you two do it again, I still have no problem. You can do it yourself. " So far, the matter was successfully resolved and the new plan was successfully issued after communication with the finance department.

\subsection{Event Two-Awkward Leadership}

Q company branch, due to the shortage of staff during the closure of the epidemic, general manager Gao asked manager Chen and manager $\mathrm{Li}$ to merge the two departments which they led. Manager Chen has strong ability and high professional quality, but has a bad temper. Manager Li is lazy and lacks responsibility. Many of her tasks end up being handled by manager Chen. Dissatisfied manager Chen looked for general manager Gao to reflect this problem, but Gao kept silent and did not contain any steps to figure out it. This went on for more than a month, and the conflict between the two women was building up until they finally broke out in a meeting.

Manager Chen raised this question again at the meeting. Due to the lack of management experience, general manager Gao was late to reach decisions and repeatedly asked manager Chen and manager $\mathrm{Li}$ for details. Manager Chen is a little angry no longer explaining, she set down a sentence directly, "You two decided." Seeing manager Chen said so, general manager Gao responded directly, "Do you still want to work?" Manager Chen replied, "I'm quitting now." Then she slammed the door and left general manager Gao shouting abuse in the meeting room. Later, the chairman intervened in this matter. He criticized general manager Gao and timely retained manager Chen. Manager Chen realized her mistake after returning, ran the opening move to apologize to general manager Gao, and then the two people harmonized. Nevertheless, this incident threw a very negative impact on general manager Gao, and she fell into an uneasy relationship with manager Chen and manager Li. She used to order takeout with them. Now she is worried that manager Chen thinks she and manager $\mathrm{Li}$ is close, or manager $\mathrm{Li}$ thinks she and manager Chen is close. At the same time, she is particularly worried about the bad relationship with the other two managers. Every time she talks with them carefully, she even does something beyond the principle to curry favor deliberately. As the top leader, she is very passive now.

\section{CASE ANALYSIS}

\subsection{Influencing Results of Leadership Silence}

Leadership silence has both positive and negative views. Appropriate use of silence strategy can assist leaders in daily management, maintain organizational harmony and shape the image of leaders. If not applied in the right way, it will cause negative emotions of subordinates, affect the relationship between superiors and subordinates and have adverse effects. 


\subsubsection{Helps Build Leadership}

For leaders with rich management experience, silence is an important means for them to build their own dignity, maintain their leadership authority, and protect themselves, such as event one. The chairman is not easy to express his opinions when middle managers have different opinions. Her silence, out of a sense of her own motive, can keep subordinates from despising her or lying to her.

\subsubsection{Helps maintain Organizational Harmony}

There will inevitably be contradictions or differences of opinions among employees. Sometimes leadership silence is more conducive to solve problems than leadership participation, such as event one. In the face of the chairman's silence, the two managers actively break the boundaries of their thinking and actively communicate with each other. If the chairman directly expressed her own views and supported Wang, it might cause an emotional blow to Liu, which would affect the relationship between the three people and hinder the organizational harmony and team cooperation.

\subsubsection{Reduces Enterprise Personnel Changes}

Silence helps to maintain organizational fairness and keeps talents for enterprises, such as event one. The two managers have been unable to achieve a colony because of the dissension over the carrying out of the new policy. In this case, one side is bound to fall into a quagmire of emotion. The chairman shows support to either side, from the other side aspect is a kind of favoritism. At the least, it will affect future work efficiency and reduce work enthusiasm. At the worst, it will cause employees to leave the enterprise and lead to brain drain. Such silence is conducive to the management of the mainstay of the enterprise and the better utilization of talents.

\subsubsection{Affects the Relationship between Superiors and Subordinates}

Leadership silence affects the solution of things, the outcome of things affects the relationship between superior and subordinate, such as event two. This matter broke the original friendly relationship between general manager Gao, manager Chen and manager Li, especially Gao is the most awkward. Moreover, it is an established fact that general manager Gao and manager Chen have had a dispute. What they say is hard to come by, and it is inevitable that they will leave hurt in each other's hearts and affect their relationship. At the same time, this issue also had an impact on the relationship between general manager Gao and the other two junior managers. She became careful in going along with them and even did something against the principles to please them, which had a negative impact on her leadership image, the relationship between superiors and subordinates, and the communication between organizations.

\subsubsection{Causes negative emotions in subordinates}

When subordinates ask for help from the leadership, they want the support of the leadership most. The leader's silence at this time is a blow to the enthusiasm of the employees such as event two. When manager Chen came to general manager Gao privately for the first time to complain about the problems between her and manager Li, it was obvious that she had been treated unfairly. She hoped to seek justice from the leader, but general manager Gao chose to keep silent. Manager Chen thought general manager Gao was partial to manager $\mathrm{Li}$, and then transferred the original dissatisfaction to Gao.

\subsection{Influencing Factors of Leadership Silence}

Leadership silence is closely related to the leader's factors and situational factors, among which the leader's factors are reflected in leader's management experience and ability level, while situational factors are reflected in employee maturity and problematic nature.

\subsubsection{Leader's Management Experience}

The chairman's silence effectively avoids the possible negative influence after the expression of opinions, maintains the harmony of the organization, and promotes team cooperation. This is because the chairman has a high-level management quality and knows how to be silent at the right time. When faced with the role of a judge, she can subtly avoid the situation that requires decision by silence, without affecting the relationship and solving the problem smoothly. On the contrary, in the case of insufficient management experience of the leader, silence is an escape from the problem and the inability to solve the problem, such as event two. General manager Gao is not clean when to be silent and when not be silent. In the final analysis, this was due to her lack of management experience. Even in the face of some very common management problems, it is easy to think about, could not make a decision in time, thus delaying the best opportunity to solve the problem.

\subsubsection{Ability Level}

The refinement of leadership ability is not overnight, but in the management practice, constantly accumulate experience, quantitative change to qualitative change, and ultimately achieve the improvement of ability. However, there are also some leaders who do not know how to improve themselves and have limited leadership ability, such as event two. General manager Gao's delay in making a decision is determined by her ability level. 
A good leader is always born with some good qualities, and leadership qualities are closely related to the success of a leader. As the general manager of the branch company due to her relationship, she lacks management experience, but she depends on her subordinates all the time, never takes the initiative to learn, and hardly improves her ability.

\subsubsection{Employee Maturity}

Leadership silence affects employee behavior, but employees in turn affect leadership silence. Employee maturity here refers to the level of capability of employees. In event one, the chairman's silence, is a test of the two managers, to test their initiative, to test whether they can be reused. It also implies trust in them; the chairman believes they will handle things well. If the protagonists of this matter are not two managers, then the matter may turn out differently. They may not mention it, which not only affects the normal operation of the company, but also causes a waste of resources.

\subsubsection{Problematic Nature}

The silence of the leader is affected by the nature of the problem itself. Generally speaking, if it is common and easy to make decisions, the leader will make decisions on the spot to reply the subordinates. When it comes to things that require long-term consideration and are not urgent, leaders are silent. In event one, the chairman cannot make a decision on the spot only by the words of manager Wang or manager Liu. The issuance of a policy requires the measurement of many factors, and all of these factors require investigation. In the face of major decisions, leaders should not make decisions hastily. It is the best choice to remain silent first, which is closely related to the nature of the event itself.

\section{CONCLUSION}

\subsection{Analysis Conclusion}

According to the research, leadership silence is ubiquitous in $\mathrm{Q}$ company, which can have positive effects in three aspects: leadership image, organizational harmony and personnel changes, and negative effects in two aspects: the relationship between superiors and subordinates and employee emotions. Leadership silence is influenced by a variety of factors. The more experienced leaders are in management, the higher their ability level is, and the better they are at using silence as a management means to assist daily management activities. The higher the maturity of employees, the easier it is for leaders to be silent to express their trust in employees or test their abilities. The nature of the issue itself can also influence the silence of the leader. Leaders often choose to remain silent on issues that require long-term consideration and are of great importance. The details are shown below.

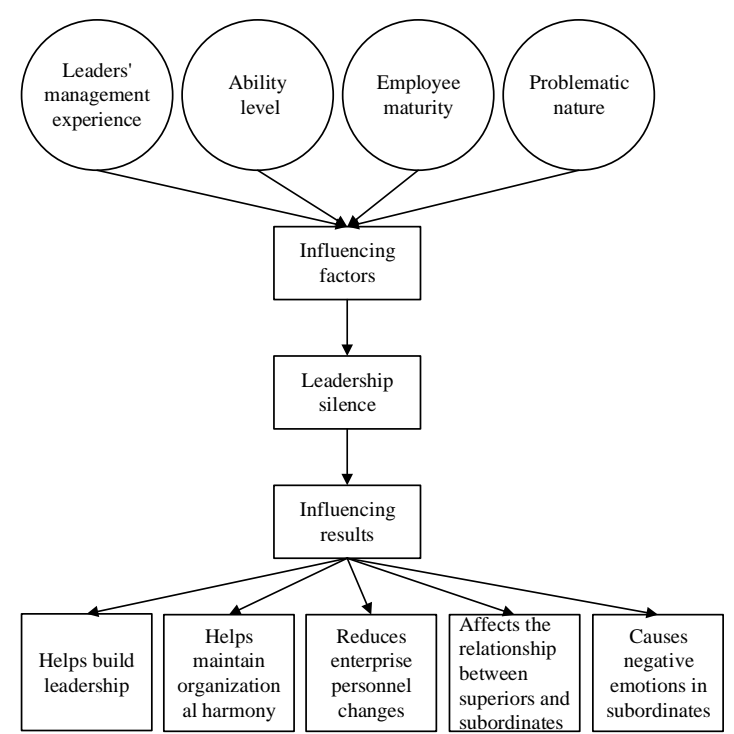

Figure 1 The influencing factors and results of leadership silence

\subsection{Management implication}

In order to make better use of leadership silence and avoid the possible adverse effects of leadership silence, this study puts forward the following suggestions. Foremost of all, enterprises should correctly understand leadership silence, should not blindly advocate or completely abandon. Objective evaluation is the premise of flexible use of leadership silence and an important guarantee to improve management efficiency and give full play to leadership. Secondly, enterprises should pay attention to the selection and training of leaders. In the selection of leaders, enterprises should conduct multiple assessments, and role-play the candidates through quality assessment questions or silent situations, so as to eliminate potential silent candidates and candidates with poor sense of responsibility. In daily operation, the enterprise should regularly hold leadership training to improve the overall quality and business ability of the leader. Thirdly, the enterprise should create a fair and tolerant corporate culture, form a loose, caring, trusting and respectful working environment, and strive to promote and create a healthy atmosphere of " treating everything as it is, not the person " in the enterprise. Finally, leaders should use leadership silence in a timely, appropriate and limited manner, and use silence flexibly according to individual differences and different leadership situations. Silence should never be used as an excuse to avoid problems and delay solutions.

\subsection{Research Limitation}

Firstly, this study only selects Q Company as a single enterprise to carry on the exploratory research to the leadership silence, the sample representativeness is 
deficient. Secondly, this study only makes an exploratory discussion on the influencing factors and results of leadership silence in Q Company, lacking of in-depth research. Leadership silence in real management practice has many factors and results. Finally, due to the limitations of qualitative research methods, the reliability of research conclusions needs further verification.

\subsection{Research Outlook}

Firstly, the follow-up research can increase the sample sampling range, so as to test whether the conclusion of a single case study is universal. Secondly, the follow-up research can carry out in-depth discussion on the factors that influence the silence of leaders, and pay attention to the specific situation and boundary conditions of the occurrence of the silence of leaders, so as to reveal the silence behavior of leaders more comprehensively. Finally, the follow-up research can explore the mechanism of leadership silence and construct theory through empirical research.

\section{REFERENCES}

[1] Huang Gui, Fu Chunguang, Tan meng jie, A probe into the Silent Behavior of Enterprise Leaders. Academic Research, vol. 07, 2013, pp. 70-78. DOI: https://doi.org/10.3969/j.issn.10007326.2013.07.011

[2] E.W. Morrison, F.J. Milliken, Organizational Silence: A Barrier to Change and Development in a Pluralistic World. Academy of Management Review, vol. 25, 2000, pp. 706-725. DOI: https://doi.org/10.5465/AMR.2000.3707697
[3] G.G. Pinder, H.P. Harlos, Employee Silence: Quiescense and Acquiescence as Responses to Perceived Injustice. Research in Personnel and Human Resource Management, vol. 20, 2001, pp. 331-369. DOI: https://doi.org/10.1016/S07427301(01)20007-3

[4] L.V. Dyne, S. Ang, I.C. Botero, Conceptualizing Employee Silence and Employee Voice as Multidimensional Constructs. Journal of Managerial Studies, vol. 09, 2003, pp.1359-1392. DOI: https://doi.org/10.1111/1467-6486.00384

[5] Liu Jun, Chen Linwei, Daiwei, A study on why leaders choose silence-self-efficacy and psychological security mechanism. Academic Research, vol. 09, 2015, pp.81-88. DOI: CNKI:SUN:XSYJ.0.2015-09-013

[6] Huang Gui, Fu Chunguang, Guan Xinhua, Construction and Measurement of the Dimension of Leadership Silence in Organization. Journal of Management World, vol.07, 2015, pp. 122129.DOI: CNKI:SUN:GLSJ.0.2015-07-015

[7] Huang Gui, Xu Zhenyi, Wang Sitong, et al., A study on the relationship between leadership silence and employee's upward understanding. Academic Research, vol.06, 2018, pp. 83-91.DOI: CNKI:SUN:XSYJ.0.2018-06-012 